\title{
Cough in spinal cord injured patients: the relationship between motor level and peak expiratory flow
}

\author{
Amy Y Wang ${ }^{1}$, Robert J Jaeger ${ }^{2}$, Gary M Yarkony ${ }^{2,3,4}$ and Rose M Turba ${ }^{5}$ \\ ${ }^{1}$ Northwestern University Medical School, Chicago, IL; ${ }^{2}$ Pritzker Institute of Medical Engineering, Illinois Institute \\ of Technology, Chicago, IL; ${ }^{3}$ Schwab Rehabilitation Hospital, Chicago, IL; ${ }^{4}$ Department of Surgery, Section of \\ Orthopaedics and Rehabilitation Medicine, University of Chicago Medical Center, Chicago, IL; ${ }^{5}$ Rehabilitation \\ Physicians of Northeast Wisconsin, Green Bay, WI, USA
}

\begin{abstract}
Pulmonary complications remain a major cause of morbidity and mortality in patients with higher level spinal cord injury. ${ }^{1-3}$ Neurologically intact individuals can cough in order to clear their air passage of lung secretions and foreign material. Patients with higher level cord injuries, with paralysis of the trunk and abdominal muscles, may not have the ability to generate an effective cough. If coughing is dependent on a caregiver, these patients will cough with reduced frequency. Because the innervation to the muscles involved in cough consists of nerves that arise from varying levels of the spinal cord, some patients with spinal cord injury have partial control over these muscles and thus can cough, though with reduced efficacy. Two hundred patients with varying levels of spinal cord injury were studied to determine if motor level and cough ability are correlated. Cough efficacy was measured using a peak expiratory flowmeter while patients were seated at $90^{\circ}$. We concluded that there is indeed a direct relationship between motor level and peak expiratory flow produced during coughing.
\end{abstract}

Keywords: spinal cord injuries; cough; peak expiratory flow rate; paraplegia; tetraplegia

\section{Introduction}

During normal cough, contraction of the abdominal muscles, especially while the glottis is closed, results in increased intra-abdominal and intrathoracic pressure. When the glottis opens, this pressure contributes to a rapid, forceful, high-velocity expiration of air, which helps to clear the bronchial passages. ${ }^{4}$ Chronic accumulation of fluid in the lungs, as is typically seen in patients with higher level injury, increases the probability of respiratory complications such as pneumonia, atelectasis, and respiratory failure. The air passages can be cleaned by artificial means, such as positioning, suction or manually assisted cough.,6 However, because these methods require assistance, the lungs may not be cleared frequently enough. The inability to cough voluntarily and effectively severely compromises a patient's health. ${ }^{7}$

It has been shown that pulmonary complications are related to the level of injury. This suggests that partial control of the muscles necessary for cough, which varies with motor level, proportionately improves a patient's ability to cough volitionally. However, up until now, no studies have been conducted to assess this relationship quantitatively.

Correspondence: AY Wang

\section{Methods}

\section{Subjects}

Two hundred and two subjects were initially recruited for the study from both the inpatient and outpatient populations of the Rehabilitation Institute of Chicago. Those patients on respirators, with tracheostomies, and with chest injuries were medically excluded from the study. In addition, patients wearing corsets or abdominal binders or other devices that could alter inspiratory and expiratory effort were instructed to remove them prior to study participation. Subjects gave informed consent as stipulated by the Institutional Review Board approved protocol.

Twenty-one patients were excluded, either because their data was unavailable or could not be verified (eg patient height, date of injury, level of injury), or they were uncooperative or could not follow directions. After data quality assurance procedures, 181 valid cases of the original 202 were available for full analysis.

The subjects' motor levels ranged from $\mathrm{C} 4$ to $\mathrm{L} 4$ and only subjects with ASIA (Frankel) grades A, B, C, and $\mathrm{D}$ were included. Motor level was determined from neurological screening tests, information provided by the patients' physicians, or subjects' medical records. Eighty-five percent of the subjects were male and $15 \%$ were female. Subjects' ages ranged from 18 to 76 years, with a median age of 34 years. Ten 
percent of the subjects were less than 1 year postinjury, and $54 \%$ were less than 5 years post-injury. The distribution of patients according to Frankel grade is shown in Figure 1.

\section{Equipment}

Peak expiratory flow during cough $^{8}$ was measured in liters per minute (Assess Peak Flow Meter, Healthscan Products, Cedar Grove, NJ). The performance of this device has recently been documented. ${ }^{9}$ It records PEF values between 100 and 900 liters/minute and can be read to an accuracy of approximately 5 liters/minute.

\section{Protocol}

Volitional coughs were measured while subjects were sitting upright at $90^{\circ}$ for five repetitions each. The sitting position was chosen because of difficulty or inability with standing and time and space constraints within the clinical environment. At least $10 \mathrm{~s}$ and up to 1 min of rest were given between coughs, depending on patient ability and level of comfort.

\section{Statistical analysis}

Standard statistical procedures were used to analyze data. Mean, linear regression, and correlation studies were performed using SPSS/PC.

\section{Results}

The mean for the five volitional coughs was computed for each subject. The means and standard deviations for peak flow at each motor level are given in Table 1.

Mean peak expiratory flows were plotted against motor level. The regression line generated (Figure 2) had a correlation of $r=0.4644$. The resulting equation was:

peak flow $=(13.0 *$ motor level $)+220$.

\section{DISTRIBUTION OF FRANKEL GRADE}

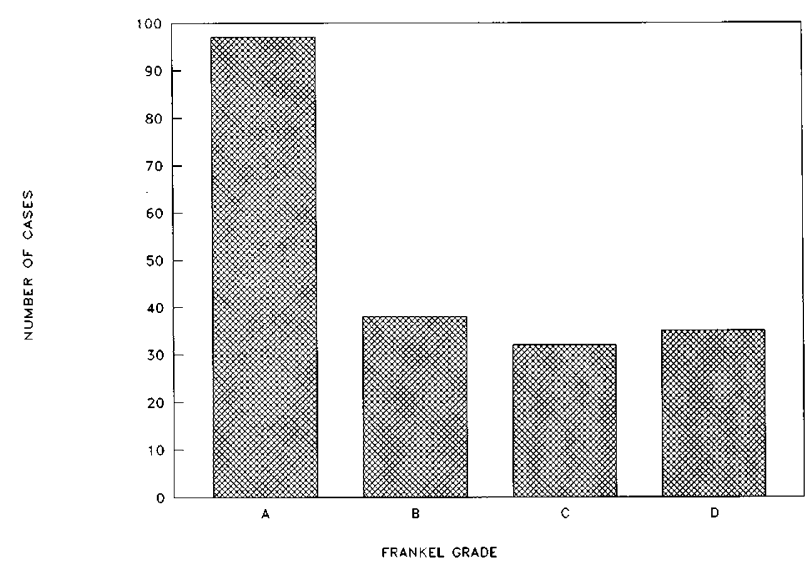

Figure 1 Distribution of Frankel grade
Both slope and intercept were significantly different from zero $(P<0.01)$. When only Frankel A subjects were included in the regression analysis (Figure 3 ), $r$ increased to 0.5581 , and the resulting equation was:

$$
\text { peak flow }=(17.5 * \text { motor level })+159 .
$$

This difference is likely due to the fact that Frankel A subjects have a more uniform completeness of injury.

A correlation analysis was performed. The correlation coefficient was greatest between peak flow during

Table 1 Mean peak expiratory flow rate, standard deviation, and number of subjects for each motor level

\begin{tabular}{lccrr}
\hline $\begin{array}{l}\text { Motor } \\
\text { level }\end{array}$ & $\begin{array}{c}\text { Numeric } \\
\text { code }\end{array}$ & $\begin{array}{c}\text { Mean } \\
(\mathrm{L} / \mathrm{m})\end{array}$ & $\begin{array}{c}S D \\
(\mathrm{~L} / \mathrm{m})\end{array}$ & $N$ \\
\hline C4 & 4 & 171.5556 & 80.7637 & 9 \\
C5 & 5 & 280.5909 & 117.9163 & 22 \\
C6 & 6 & 297.0000 & 107.9280 & 19 \\
C7 & 7 & 354.7105 & 116.2583 & 38 \\
C8 & 8 & 319.4286 & 103.6579 & 7 \\
T1 & 9 & 549.0000 & .0000 & 1 \\
T2 & 10 & 378.3333 & 79.9771 & 3 \\
T3 & 11 & 333.0000 & 66.4342 & 5 \\
T4 & 12 & 355.4000 & 148.6765 & 10 \\
T5 & 13 & 322.5000 & 225.5671 & 2 \\
T6 & 14 & 369.5455 & 180.7509 & 11 \\
T7 & 15 & 494.5714 & 198.8072 & 7 \\
T8 & 16 & 422.0000 & 153.5985 & 5 \\
T9 & 17 & 386.8000 & 138.6279 & 5 \\
T10 & 18 & 397.3571 & 159.5897 & 14 \\
T11 & 19 & 659.0000 & .0000 & 1 \\
T12 & 20 & 440.7143 & 213.3446 & 7 \\
L1 & 21 & 550.6000 & 251.2604 & 5 \\
L2 & 22 & 526.3333 & 217.7096 & 6 \\
L3 & 23 & 670.6667 & 118.8122 & 3 \\
L4 & 24 & 396.0000 & .0000 & 1 \\
\hline
\end{tabular}

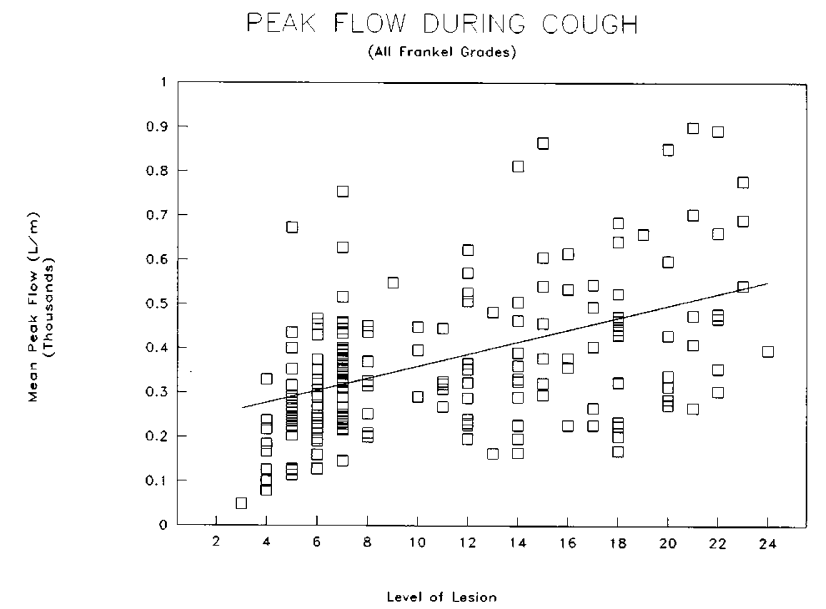

Figure 2 Plot of peak flow versus motor level for data with all subjects. Linear regression line fit to the data has a slope of 13.0 and intercept of 220 


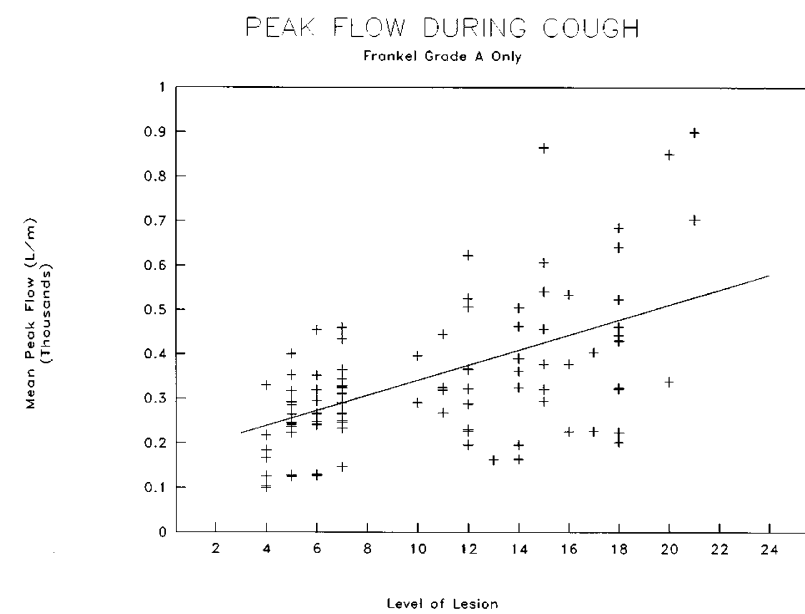

Figure 3 Peak flow versus motor level for Frankel grade A only. Linear regression line has slope of 17.5 and intercept of 159

cough and motor level. Other variables, such as ASIA (Frankel) grade (0.1810), height (0.0836), weight $(0.1350)$, age $(-0.0795)$, and years post-injury $(0.0720)$ did not have statistically significant correlation coefficients with peak flow $(P<0.001)$.

This data was compared to existing data on the incidence of pulmonary complications as a function of level of injury. As expected, complications tend to be greatest in higher motor levels. Conversely, complications appear to be lower in those with lower motor levels.

\section{Conclusions}

There was a statistically significant correlation between peak expiratory flow and motor level. The relationship between these two quantities was reasonably approximated by a first-order linear regression model. It could be argued that a strictly linear model is not appropriate here since one might expect peak flow to approach an asymptote of neurologically intact values. However, in neurologically intact individuals, peak flow during cough is a function of height and age. The exact nature of the transition from the spinal cord injured to neurologically intact state with respect to respiratory function as motor level decreases remains problematic. We therefore elected to use the simplest possible model in our initial approach to the problem. In addition, in the region of cervical and high thoracic injuries, the relationship between motor level and peak flow might in fact be close to linear.

There may be several potential sources of bias in this study. The nature of informed consent may preclude obtaining a truly randomized sample of the spinal cord injured population, because those patients refusing to participate may have respiratory complications, making coughing through a tube seem extremely unpleasant. Similarly, the majority of subjects studied were patients in an outpatient clinic. Patients who regularly make their scheduled appointments are likely to be those who manage their health most conscientiously. Some error probably exists determining motor level, because the large number of subjects in this study made it impossible to have a neurologist perform a detailed examination of motor function. In addition, information obtained from subjects' medical records may not reflect the subjects' neural function at the actual time of study. After a spinal cord injury, it is not uncommon for patients to regain one to three levels of motor function or improve Frankel grade by one or two grades, possibly increasing peak expiratory flow during cough. ${ }^{3}$ The level of cough measured is also highly dependent on individual effort and current state of health. This is shown by the data in Figure 2, showing that the range of PEF's produced varied widely among subjects with lower lesions, possibly because of differences in effort. However, the range was notably narrower in subjects with cervical lesions, whose variations may result primarily from differences in ability and Frankel grade.

The data reported in this study support the hypothesis that peak expiratory flow during cough is a function of motor level. However, whether PEF produced during coughing correlates with actual mobilization and clearing of secretions remains to be seen. Other factors, such as peak excitatory force or forced vital capacity, may also contribute to cough efficacy. Cough must be a fundamental part of the rehabilitation program in spinal cord injured patients. An understanding of how a patient's motor level affects cough could be important in teaching patients how to maximize use of those muscles that remain under their control, such as the accessory muscles of respiration. ${ }^{10}$ This could then be used to help them clear their lungs of secretions, perhaps in combination with manually assisted cough ${ }^{6}$ or electrical stimulation of abdominal muscles. ${ }^{11}$ These findings can serve as a standard for determining the efficacy of these methods of improving cough. It will be possible to predict how much cough ability patients who initially present with a given injury and resulting motor level and Frankel grade will retain or eventually recover. Thus, therapy can be instituted immediately to try to minimize pulmonary complications during the acute post-injury period and prevent respiratory muscle atrophy. It is hoped that a better understanding of cough will help identify those at greatest risk for respiratory compromise and reduce the incidence and severity of pulmonary complications in patients with spinal injuries.

\section{Acknowledgements}

The authors wish to thank the staff of the Rehabilitation Institute of Chicago for providing the equipment required and helping to recruit patients for the study. This work was supported in part by NIDDR (H133N0008). 


\section{References}

1 Polatty RC, McElaney MA, Marcelino V. Pulmonary complications in the spinal cord injury patient. Physical Medicine and Rehabilitation: State of the Art Reviews 1987; 1: $353-373$.

2 Reines HD, Harris RC. Pulmonary complications of acute spinal cord injuries. Neurosurgery 1987; 21: $193-196$.

3 Stover SL, Fine PR. Spinal Cord Injury: The Facts and Figures. Birmingham Alabama, University of Alabama Birmingham Press, 1986.

4 Jones JG, Clarke SW. Dynamics of Cough. Br J Anaesth 1970; 42: $280-285$

5 Braun SR, Giovannoni R, O'Connor M. Improving the cough in patients with spinal cord injury. Am J Phys Med 1984; 63: 1 - 10 .

6 Kirby NA, Barnerias MJ, Siebens AA. An evaluation of assisted cough in quadriparetic patients. Arch Phys Med Rehabil 1966; 47: $705-710$.

7 Traver GA. Ineffective airway clearance: physiology and clinical application. Dimensions of Critical Care Nursing 1985; 4: $198-$ 208.

8 Leiner GC, Abramowitz S, Small MJ, Stenby VB. Cough peak flow rate. Am J Med Sci 1966; 251: $211-214$.

9 Shapiro SM et al. An evaluation of the accuracy of Assess and Miniwright peak flowmeters. Chest 1991; 99: 358-362.

10 Estenne M, De Troyer A. Cough in tetraplegic subjects: an active process. Ann Intern Med 1990; 112: 22 - 28.

11 Yarkony GM, Roth EJ, Cybulski GR, Jaeger RJ. Applications of neuromuscular stimulation in spinal cord injury. Part I: Restoration of functional movement of the extremities. Part II: Prevention of secondary complications. Arch Phys Med Rehabil 1992; 73: $78-86,195-200$ 